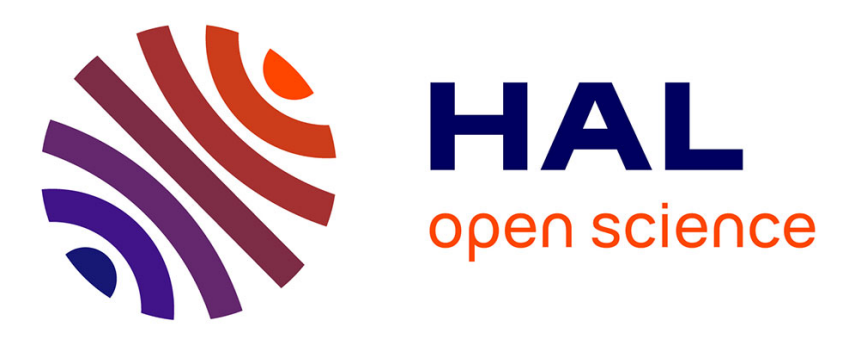

\title{
Nouvelle méthode expérimentale d'étude du comportement dynamique à grande vitesse de déformation des plaques minces - sollicitation par explosion
}

O. Pennetier, J. Renard

\section{To cite this version:}

O. Pennetier, J. Renard. Nouvelle méthode expérimentale d'étude du comportement dynamique à grande vitesse de déformation des plaques minces - sollicitation par explosion. Journal de Physique IV Proceedings, 1997, 07 (C3), pp.C3-131-C3-136. 10.1051/jp4:1997325 . jpa-00255482

\author{
HAL Id: jpa-00255482 \\ https://hal.science/jpa-00255482
}

Submitted on 1 Jan 1997

HAL is a multi-disciplinary open access archive for the deposit and dissemination of scientific research documents, whether they are published or not. The documents may come from teaching and research institutions in France or abroad, or from public or private research centers.
L'archive ouverte pluridisciplinaire HAL, est destinée au dépôt et à la diffusion de documents scientifiques de niveau recherche, publiés ou non, émanant des établissements d'enseignement et de recherche français ou étrangers, des laboratoires publics ou privés. 


\title{
Nouvelle méthode expérimentale d'étude du comportement dynamique à grande vitesse de déformation des plaques minces - sollicitation par explosion
}

\author{
O. Pennetier et J. Renard
}

Laboratoire Énergétique, Explosions, Structures (LEES), Université d'Orléans, 63 avenue de Lattre de Tassigny, 18020 Bourges cedex, France

\begin{abstract}
Résumé : Au travers de cet article est décrite une méthode expérimentale originale permettant d'obtenir les champs de déformation et de déplacement d'une plaque métallique mince. La sollicitation est apportée par la détonation d'un mélange gazeux et le champ de pression induit, transitoire et de forte intensité, engendre de grands déplacements. Après une description détaillée de la méthode, les résultats obtenus pour un cas test sont présentés. On peut notamment suivre l'évolution de la plastification de la plaque, en fonction de l'espace et du temps. Ces résultats peuvent être repris et confrontés avec des programmes numériques, ce qui permet de caler les paramètres viscoplastiques sous haute vitesse de déformation.
\end{abstract}

\begin{abstract}
In this paper is described an original and experimental method in order to obtain both fields of displacement and strain, using a thin metallic plate. The loading is generated by the detonation of a gazeous mixture. The pressure induced, transient and of high intensity, leads the plate to large displacements. After an accurate description of the experimental set-up, main results are presented. We can particularly follow the plastic waves, using both time and space description. Those results can be introduced in a numerical code, which can permit numericians to check viscoplastic laws parameters under high strain rates.
\end{abstract}

\section{INTRODUCTION}

Le comportement mécanique des matériaux sous une vitesse de déformation élevée est un domaine dans lequel les études expérimentales se limitent souvent à la mesure des paramètres d'état à l'issue d'essais transitoires (perforation, impact). L'évolution temporelle de ces paramètres n'est principalement étudiée que de manière monodimensionnelle, avec par exemple l'essai à la barre de Hopkinson. Les mesures en dynamique rapide sur un milieu bidimensionnel, peu nombreuses dans la littérature, semblent encore nécessaires afin de préciser au mieux les coefficients des lois de comportement viscoplastique [1] (BodnerPartom, Chaboche...). Il est ici proposé d'apporter une méthode expérimentale originale d'obtention des champs de déformation et de déplacement au cours du temps, sur une structure de géométrie simple, la sollicitation résultant de la détonation d'un mélange gazeux.

\section{MONTAGE EXPERIMENTAL}

\subsection{La sollicitation}

La sollicitation est apportée par la détonation d'une sphère gazeuse en champ proche. La manipulation de tels explosifs est une opération où les mesures de sécurité doivent être scrupuleusement respectées. Néanmoins, ces manipulations restent moins dangereuses que celles d'explosifs solides.

Il existe une loi de similitude, appelée loi de Hopkinson, qui permet de transposer des cas réels aux modèles réduits [2]. Il est ainsi possible d'étudier des cas réels d'explosions en se servant de modèles à échelle réduite [3]. 


\subsubsection{Notions de détonique et caractéristiques du champ de pression}

Le profil de pression obtenu, transitoire, a comme particularité d'être déterministe, et donc reproductible dans les expériences [4]. L'onde de choc induite, de forme sphérique, a déjà fait l'objet de plusieurs études ; la mise en oeuvre expérimentale et le profil de pression résultant sont parfaitement maîtrisés.

Une détonation peut se caractériser par sa distance réduite $\lambda$, rapport entre $\mathrm{d}_{\mathrm{i}}$, la distance du centre de l'explosion à la plaque, et la racine cubique de l'énergie du gaz considéré.

$$
\begin{aligned}
\lambda=\frac{d_{i}}{\sqrt[3]{E_{0}}}, \text { avec } E_{o}=\frac{4}{3} \pi\left(R_{\text {bulle }}\right)^{3} E_{v} \\
\text { et } E_{v}: \text { énergie spécifique du milieu réactif. }
\end{aligned}
$$

Une détonation possède un profil de pression type (figure 1). Cette pression étant liée à la présence de la structure dans le champ de la détonation, est une pression réfléchie. En un point considéré, on distingue quatre phases distinctes successives; un temps d'arrivée $t_{\mathrm{a}}$, fonction de la célérité de l'onde sur le trajet, une discontinuité de pression (temps de montée $<3 \mu \mathrm{s}$ ) où l'on passe d'une pression relative nulle à la surpression maximale $\Delta \mathrm{p}^{+}$, une durée $\mathrm{t}^{+}$, pendant laquelle le signal de pression décroît rapidement (période de surpression) et une durée $t^{-}$, où l'on observe une dépression.

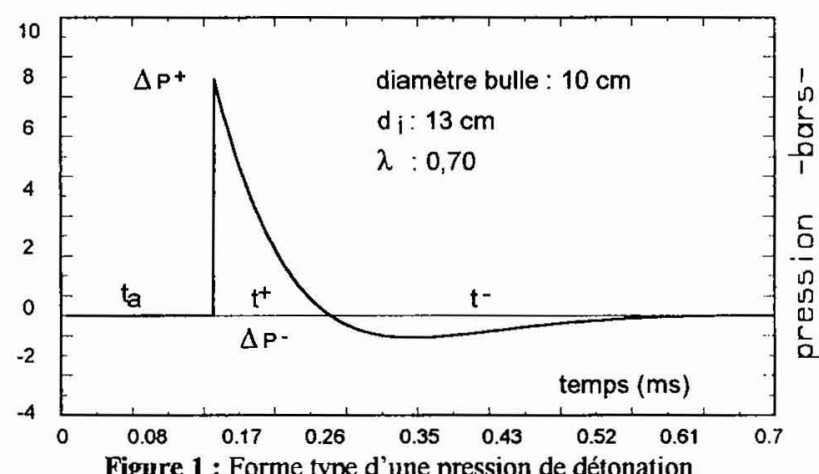

Figure 1 : Forme type d'une pression de détonation

\subsubsection{Formulation analytique du champ de pression}

L'expression générale du profil spatio-temporel de la pression peut être approchée analytiquement [5], [6], et a pour expression générale, en prenant l'origine des temps en $\mathrm{t}_{\mathrm{a}}$ :

$$
\mathrm{p}(\mathrm{t})=\Delta \mathrm{p}^{+} \frac{\sin \left(\frac{\pi}{\mathrm{t}^{-}}\left(\mathrm{t}-\mathrm{t}^{+}\right)\right)}{\sin \left(-\pi \mathrm{t}^{+} / \mathrm{t}^{-}\right)} \mathrm{e}^{-\mathrm{tK} / \mathrm{t}^{+}} \text {, pour } 0<\mathrm{t}<\left(\mathrm{t}^{+}+\mathrm{t}^{-}\right)
$$

Les termes de cette formule peuvent s'exprimer à l'aide de développements polynomiaux, estimés au moyen de nombreuses expériences [7]. On obtient la pression de l'onde réfléchie, pour la détonation d'un gaz considéré. Cette relation, validée par de nombreuses expériences, sert de référence pour notre étude. Le profil type de pression spatio-temporelle de la détonation d'un mélange propane-oxygène est présenté ci-contre, figure 2 . Le rapport $\mathrm{r} / \mathrm{d}$ correspond à la position du profil de pression par rapport au rayon de la plaque. La pression est présentée en cinq points de la plaque circulaire.

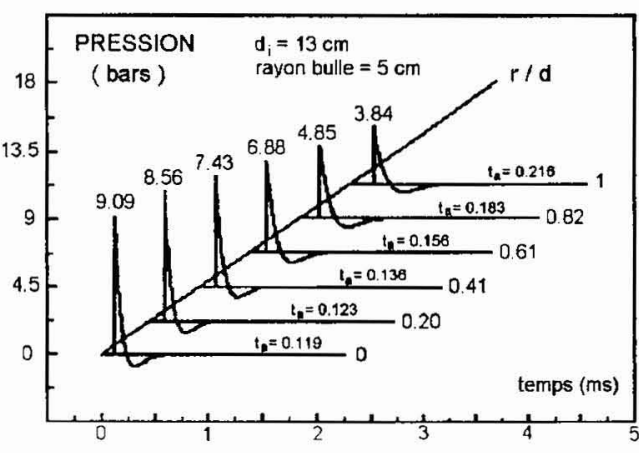

Figure 2 : Répartition spatio-temporelle de la pression 


\subsection{Nature du matériau testé}

\subsection{Caractéristiques mécaniques}

Les plaques utilisées sont constituées d'un alliage d'aluminium 5754 (AlMg3, état $\mathrm{H} 111$ ), dont les caractéristiques sont les suivantes :

- Masse volumique $\rho: 2670 \mathrm{Kg} / \mathrm{m}^{3}$

- Coefficient de Poisson v : 0.30

- Module d'Young E : $72000 \mathrm{MPa}$

La masse volumique a été confirmée par pesée et le module d'Young a été déterminé par mesures dynamiques sur le premier mode de vibration, en considérant des plaques de géométries diverses. La limite élastique d'un matériau a comme particularité d'évoluer en fonction de la vitesse de déformation. Pour une vitesse faible (inférieure à $10^{-5} \mathrm{~s}^{-1}$ ), on peut estimer, par essais de traction, cette limite élastique $\sigma_{0}$ à 102 $\mathrm{MPa}$, ce qui correspond bien à ce type d'alliage. L'évolution de cette limite élastique peut être approximée, en négligeant l'écrouissage, par une loi viscoplastique de Perzyna, en prenant pour coefficients un exposant $\mathrm{n}=4$, et un coefficient $\gamma$ de $6500 \mathrm{~s}^{-1}$, avec la relation (3).

$$
\sigma_{\mathrm{e}}=\sigma_{\mathrm{o}}\left(1+\frac{\dot{\varepsilon} \operatorname{sgn} \sigma}{\gamma}\right)^{(1 / \mathrm{n})}
$$

\subsubsection{Caractéristiques géométriques}

La plaque, circulaire, a pour épaisseur $0.5 \mathrm{~mm}$ et son diamètre est de $26.6 \mathrm{~cm}$. Afin d'obtenir une apparition rapide des non linéarités et d'assurer une bonne tenue mécanique face à une sollicitation importante, la plaque a été encastrée sur sa périphérie.

\subsection{Montage et déroulement de l'expérience}

La plaque testée est encastrée entre deux anneaux massifs, eux-mêmes fixés sur un bâti plan de grandes dimensions supposé indéformable (figure 3). La détonation est initiée par la vaporisation sous haute tension d'un fil de cuivre fin. L'onde de choc lancée vient frapper la plaque avec une célérité comprise entre 600 et $1000 \mathrm{~m} / \mathrm{s}$ et provoque une déformation plastique brutale du matériau.

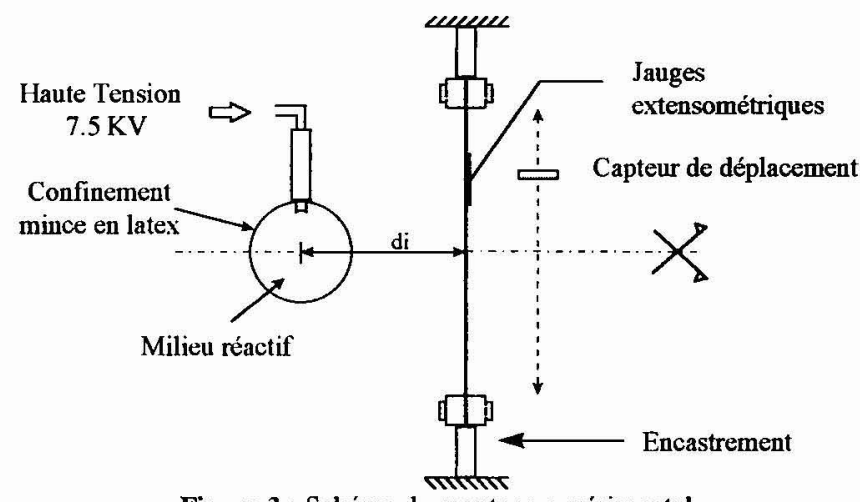

Figure 3 : Schéma du montage expérimental 
L'évolution de la structure est suivie, dans le temps et l'espace, par différents appareils de mesure dont la description est précisée au paragraphe suivant. Les signaux sont enregistrés sur une chaîne d'oscilloscopes numériques, et transférés par interface IEEE sur PC. Les différents fichiers sont traités en temps différé.

\section{INSTRUMENTATION DE LA PLAQUE}

La chaîne de mesures, ainsi que celle d'acquisition, doit être compatible avec la nature transitoire des signaux. Les différents appareils utilisés, ainsi que leurs caractéristiques, sont précisés dans le tableau $n^{\circ} 1$.

Tableau $n^{\circ} 1$ : métrologie employée

\begin{tabular}{|c|c|c|c|c|}
\cline { 2 - 5 } \multicolumn{1}{c|}{} & Type & Marque & Mesure & Caractéristiques \\
\hline Pression & Piézo-électrique & Kistler $603 B$ & $0-200 \mathrm{bars}$ & Temps de montée : $1 \mu \mathrm{s}$ \\
\hline Déplacement & Diode laser & Kevence $L B 70$ & $\begin{array}{c} \pm 40 \mathrm{~mm} \\
\text { Résolution }<180 \mu \mathrm{m}\end{array}$ & $\begin{array}{c}\text { Fréqucnce de réponse } \\
\text { maximale }: 700 \mathrm{~Hz} .\end{array}$ \\
\hline Déformation & Jauges d'extensométrie & Kyowa KFG $10-120$ & Limite $: 2.5 \%$ & $120 \Omega-$ Longucur : $10 \mathrm{~mm}$ \\
\hline
\end{tabular}

Les jauges d'extensométrie se situent dans les directions principales de la plaque, radiale et circonférencielle, et sont notées $\mathrm{J}_{\mathrm{r}}$ et $\mathrm{J}_{\theta}$, comme indiqué sur la figure 4 . La plaque et la sollicitation étant axisymétriques, l'instrumentation d'un seul rayon de la plaque est suffisante. Les jauges tangentielles donnent directement la valeur de l'allongement dans le plan de la plaque.

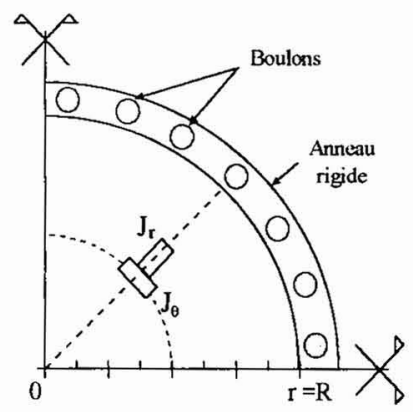

Figure 4 : Position des jauges d'extensométrie

\section{RESULTATS DES EXPERIENCES}

\subsection{Choix préliminaires}

Concernant l'intensité de la sollicitation, il a été nécessaire de déterminer une configuration optimale afin d'utiliser au mieux les capacités de notre métrologie. Notre choix s'est porté sur les paramètres suivants

- Nature du mélange gazeux : Propane - Oxygène en proportions stoechiométriques,

- Diamètre de la sphère gazeuse : $10 \mathrm{~cm}$,

- Distance $\mathrm{d}_{\mathrm{i}}: 13 \mathrm{~cm}$.

Le choix du mélange gazeux est justifié par l'énergie volumique importante dégagée par la réaction chimique de détonation (Energie volumique libérée à $20^{\circ} \mathrm{c}: 14.16 \mathrm{MJ} / \mathrm{m}^{3}$, équivalent TNT $=5$ ). Le couple de paramètres $\left(\mathrm{d}_{i}\right.$, diamètre bulle) entraîne une libération d'énergie suffisante pour provoquer une déformation plastique du matériau de l'ordre de $2 \%$, valeur compatible avec les jauges résistives employées. Cette configuration a été conservée pour l'ensemble des résultats présentés ci-après. 


\subsection{Résultats de la campagne d'essais}

La première chose à vérifier a été la reproductibilité des signaux. La figure 5 présente le résultat de la déformation au centre de la plaque en fonction du temps, pour deux expériences similaires. La bonne corrélation entre ces deux courbes prouve la reproductibilité des expériences. Il n'est donc pas nécessaire d'instrumenter la totalité de la plaque, plusieurs expériences réalisées dans des conditions identiques permettant de reconstituer une seule et unique expérience.

Cette figure nous montre une forte discontinuité de la déformation, qui traduit un écoulement plastique brutal du matériau. En intégrant numériquement ce même signal, il est possible de visualiser l'évolution temporelle de la vitesse de déformation du point central de la plaque (figure 6). On voit ainsi clairement des vitesses de déformation assez élevées, avec un pic de $300 \mathrm{~s}^{-1}$.

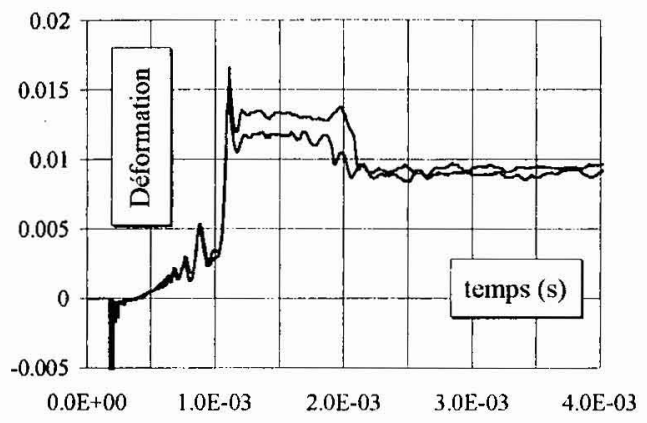

Figure 5 : Evolution temporelle de la déformation au centre de la plaque

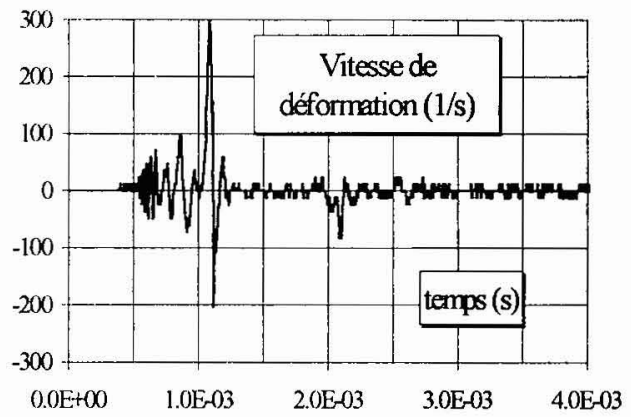

Figure 6 : Evolution temporelle de la vitesse de déformation au centre de la plaque

Les capteurs de déplacement et de déformation sont disposés le long de la plaque et les enregistrements permettent de reconstituer l'histoire globale de la réponse, après interpolation (figures 7). On peut ainsi suivre la progression des ondes plastiques sur la surface de la plaque. Au cours de cet essai, la célérité de l'onde de choc est de $600 \mathrm{~m} / \mathrm{s}$. Les parties grisées correspondent à un dépassement du seuil de plasticité initial $\sigma_{0}$, qui ne tient pas compte de l'augmentation due à la vitesse de déformation. Les zones plastiques prennent naissance à la périphérie de la plaque, et l'écoulement plastique provoqué se propage à la vitesse de $250 \mathrm{~m} / \mathrm{s}$. Les allongements radiaux de la plaque, $u(r)$ sont directement obtenus à l'aide des jauges de déformations tangentielles, par la relation $u(r)=r . \varepsilon_{\theta}(r)$, avec un allongement nul au centre $(\mathrm{u}(0)=0)$.

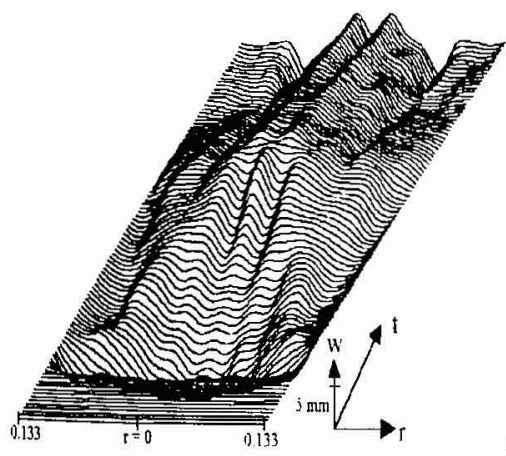

a- déformée

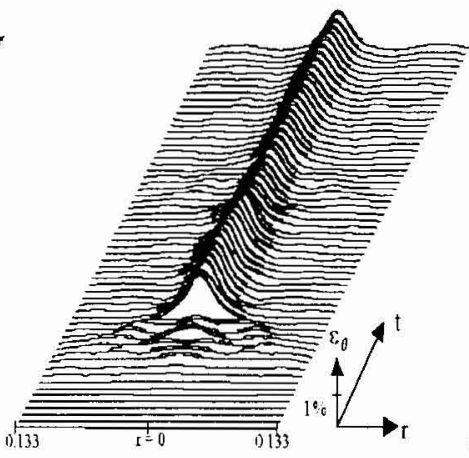

b- déformation tangentielle

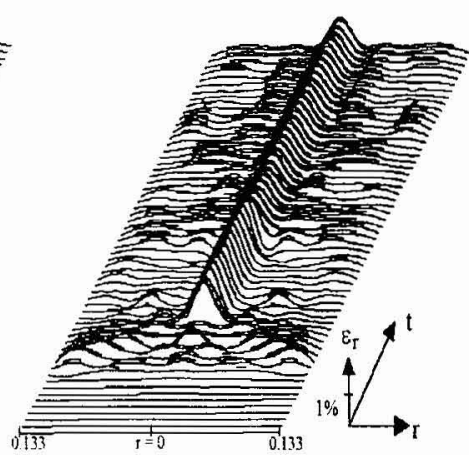

c- déformation radiale

Figure 7 : Evolutions spatio-temporelles de la plaque (durée totale d'observation : 4 ms) 


\section{REFLEXIONS SUR LA METHODE : ANALYSES ET PERSPECTIVES}

Dans la littérature, on peut citer l'exemple de la barre de Hopkinson, permettant de suivre l'évolution temporelle de la vitesse de déformation. Des signaux d'allures similaires sont obtenus, mais de nature monodimensionnelle (impacts sur des cylindres de faible diamètre). Ces essais nécessitent un appareillage relativement important, ce qui occasionne un coût de revient assez élevé, [8], comparé à celui présenté dans notre étude.

Concernant nos expériences, nous avons été limités par l'utilisation de jauges d'extensiométrie à $2.5 \%$ d'allongement, valeur atteinte assez rapidement. Des détonations beaucoup plus fortes peuvent être obtenues, et l'utilisation de jauges extensométriques adaptées permettrait d'observer des vitesses de déformations de l'ordre de $10^{4}$ à $10^{5} \mathrm{~s}^{-1}$. Ces grandeurs peuvent être atteintes sans que le support expérimental existant soit modifié. Au niveau des déplacements, il pourrait être judicieux d'utiliser un moyen optique (caméra rapide), afin de visualiser une évolution plus précise des déformées.

\section{CONCLUSION}

Les expérimentations menées en dynamique plastique sous une forte vitesse de déformation sont peu courantes dans la littérature, surtout pour les cas non monodimensionnels. Les résultats obtenus ici peuvent contribuer au calage des paramètres viscoplastiques. Dans ce domaine, plusieurs lois viscoplastiques peuvent être appliquées, mais les numériciens ont besoin d'essais pour obtenir ces valeurs. et valider ainsi leurs modèles. Les deux parties essentielles de cette expérimentation, la sollicitation et la réponse de la structure, sont bien maîtrisées expérimentalement, et les extensions à cette étude peuvent être nombreuses et intéressantes (application à d'autres matériaux, à des formes géométriques particulières, à d'autres gammes de sollicitation plus fortes ...).

\section{Références}

[1] Woznica K, Klosowski P., Weichert D., "Sur le comportement dynamique des structures dans le domaine élasto-viscoplastique", $2^{\text {nd }}$ colloque national en calcul des structures, Giens 16-19 Mai 1995 (Eds. Hermès, 1995) 2, pp. 219-224.

[2] Baker W.E., Cox P.A., Westine P.S., Kulesz J.J., Strehlow R.A., Explosions hazards and evaluation (Fundamental studies in engineering, Elsevier, Rotterdam, 1983)

[3] Renard J., Desrosier C., Bailly P., Brossard J., Réponses dynamiques de structures du génie civil. Matériaux Mécanique Electricité (1988) 427, pp 60-64.

[4] Renard J., Pennetier O., "Non linear dynamic responses of plates submitted to explosions - Numerical and experimental study", European Conf. on Struct. Dynamics, Eurodyn'96, 5-8 juin 1996, G. Augusti \& al. (Eds. A. A. Balkema, 1996) 2, pp. 689-694.

[5] Delaroche C., "Caractérisation d'une onde due à la détonation d'un mélange gazeux air - hydrocarbure en milieu libre", DER - EDF, (1983), Rapport HP/ 219 / 83 / 48.

[6] Lannoy A.,"Analyse des explosions air-hydrocarbures en milieu libre : Etudes déterministe et probabiliste du scénario d'accident. Prévision des effets de surpression", Bulletin Direction Etudes et Recherches EDF A4 (1984).

[7] Brossard J., Desrosier C., Purnomo H., Renard J., "Pressure loads on plane surface submitted to an explosion", 19th Int. Symp. on shock waves, 26-30 juin 1993, R Brun \& L.Z Dumitrescu (Eds. SpringerVerlag, 1995) 4, pp. 387-392.

[8] Bodner S.R., Symonds P.S., Experiments on viscoplastic response of circular plates, J. Mech. Phys. Solids 27 (1979) 91-113. 